Research Article

\title{
A Classification Method of Tourism English Talents Based on Feature Mining and Information Fusion Technology
}

\author{
Xin Wei $\mathbb{D}^{1,2}$ \\ ${ }^{1}$ Graduate School, Nueva Ecija University of Science and Technology, Cabanatuan, Nueva Ecija 3100, Philippines \\ ${ }^{2}$ English Language Teaching Unit, Lu'an Vocational and Technical College, Lu'an 237100, China \\ Correspondence should be addressed to Xin Wei; xin615290@lvtc.edu.cn
}

Received 26 February 2021; Revised 10 April 2021; Accepted 10 May 2021; Published 19 May 2021

Academic Editor: Muhammad Usman

Copyright (c) 2021 Xin Wei. This is an open access article distributed under the Creative Commons Attribution License, which permits unrestricted use, distribution, and reproduction in any medium, provided the original work is properly cited.

\begin{abstract}
With the rapid development of the Internet, text data has become one of the major formats of big data tourism and improves the quality and promotes the optimization and upgradation of tourism English talents. This paper proposes a model of tourism English talent resources based on data mining techniques using a big data framework. The characteristic distribution structure model is built to identify and blend the characteristics of tourism English talent resources. Connection feature mining and information fusion are combined to share data and schedule resources during the talent training process. Initially, the proposed research work uses a cloud storage system for developing intercultural communicative competence of tourism English talents. Next, the optimal scheduling design of tourism English talent training resource's big data is carried out. Finally, the fuzzy clustering method deals with the adaptive clustering of tourism English talent resource distribution big data. The simulation findings show that the proposed method has high precision and big data computation efficiency. Moreover, it can successfully mentor the development of a new framework of tourism English talent training.
\end{abstract}

\section{Introduction}

The training goal of tourism English talents is located in the technical application of the tourism industry and the compound management talents to cultivate the middle level of the tour guide industry, the hotel industry and the middle level, and the management talents above the middle level. Therefore, the training target of the Higher Vocational Tourism English talents is the technical nature of the talent type, the professionalism of knowledge ability, and the graduate's direction [1]. At present, a lot of travel agencies and hotel enterprises urgently need strong English listening, speaking, reading, writing, translation comprehensive ability, familiarity with the tourism industry-related policies, policies, good tourism, hotel management, and other professional knowledge and skills, with cross-cultural interdisciplinary talents. Tourism English majors must be closely integrated into the market because the professional orientation of the students is suitable for the needs of the current society; it depends highly on whether the orientation of the majors and the arrangement of the courses are reasonable and whether they have the current travel agency and the knowledge structure required by the hotel. Direct trade relations are established with many countries and regions. The number of the top five hundred multinational companies in the world has been increasing. Also, the number of multinational corporations and the amount of outbound tourism in the world ranks are increasing [2]. Therefore, there is a strong ability to express English, the managers who are familiar with the tourism knowledge and tour guide business, the tour guides, and the market demand. To improve tourism English, the quality of talents needs to optimize the training mode of tourism English talents and promote the improvement of the quality of tourism English talents [3]

Given the strong practical characteristics of a tourism English major, the most effective way to train its talents can only be the combination of production and learning. Therefore, in addition to strengthening the construction of training rooms, internships, outside schools, and training 
bases, big data scheduling and integration of tourism English talent resources should also be carried out according to the requirements of the quality, knowledge, and ability of social posts. The data mining methods for tourism English talents mainly include blind convolution algorithm, feature extraction method, time-frequency analysis method, and statistical analysis method. These methods cannot eliminate the convolution effect of the mining channel to singular features. There are several data mining tools that companies can use to translate raw data into actionable insights. These include statistics, artificial intelligence, hidden Markov models, metalearning, genetic algorithm, machine learning, and decision tree. It affects the accuracy of big data features mining in the distribution of big tourism English talent resources. In [4], a new information mining and scheduling algorithm for tourism English talent resources based on differential cumulate function feature mining is proposed. To improve the ability of information collection and feature analysis of tourism English talent resources, the distributed resources information of tourism English talent resources under a big data environment is fused and processed. However, the algorithm has high computational complexity and a big implementation cost [5-7].

In this research work, an effective and intelligent computational model is proposed. In view of the above problems, a new model of tourism English talents training is proposed based on big data, and it constructs a big data mining model of tourism English talents. Data mining is widely used in the field of artificial intelligence (AI), marketing, government intelligence (GI), services, and advertising. There are some other industries like crime agencies, retail healthcare, e-commerce, telecom, biological data analysis, and information retrieval like communication systems. Data mining is used to examine or explore the data using queries. These queries can be fired on the data warehouse. In the cloud storage system, the optimal scheduling design of tourism English talent training resource big data is carried out, and the fuzzy clustering method is used to deal with the adaptive clustering of tourism English talent resource distribution big data. This research work adopts the attribute distribution structure model to classify and fuse the features of tourism English talent resources, and it combines the methods of association feature mining and information fusion to share data and schedule resources in the process of talent training. Finally, the performance test is carried out through the simulation experiment, which shows the superior performance of this method in improving the big data analysis ability of tourism English talents training.

The rest of the paper is organized as follows: tourism English talent resource distribution model is explained in Section 2; big data feature extraction is discussed in Section 3, and, in Section 4, experimental results and discussion are discussed. Finally, we demonstrate a conclusion and future work in Section 5.

\section{Tourism English Talent Resource Distribution Model}

In this section, we elaborate the new cross-cultural communicative competence model that is proposed in the book titled "Intercultural Communication in Context" and the elements of cross-cultural communicative competence of tourism English talents.

2.1. The Formation of Cross-Cultural Communication Ability Elements. The formation of cross-cultural communication ability elements includes four elements, that is, knowledge factors, emotional factors, mental activity characteristics, and situational characteristics. Cross-cultural communication elements of tourism English talents are shown in Figure 1. The knowledge in the cross-cultural communication skills mentioned in the figure refers to the degree of understanding of the target's culture in the party of communication, and the cross-cultural communication ability is directly proportional to its understanding. Among them, knowledge factors include cultural values, beliefs and behaviors, verbal and nonverbal scripts, simplified and rigid cognition, and ethnocentrism. These factors can have a positive or negative impact on the communicators. Emotional factors refer to the attitudes adopted by the communicators in dealing with communicative objects from different cultural groups, that is, approaching or alienating. In the intercultural communication activities, inevitably there will be fear or anxiety. Therefore, if they are willing to communicate, the motivation of their communication is a more important emotional factor in cross-cultural communication. The ability to deal with stress and tolerance is positively related to intercultural communicative competence, and knowledge and affective factors in cross-cultural communicative competences interact with and support each other. The more knowledge, the less anxiety, and the more cross-cultural, the more arguments. The stronger the communicative motivation is, the stronger the communicative motivation is, and the more chances of obtaining relevant experience are, the more intercultural communication knowledge becomes more and more abundant.

Similarly, mental activity factors are a comprehensive manifestation of knowledge and emotional factors, including verbal and nonverbal communication and role play. Having more opportunities to practice using the target language is an important factor to enhance their abilities, and attention should also be paid to a series of auxiliary nonlanguage symbols such as body language, spatial language, and time language of the communication partner in communication. Role play is related to context. It is the use of verbal and nonverbal symbols in the destination culture according to their role identities. Moreover, for situational characteristics, the environment has a great influence on both sides of the communication. Situational characteristics 


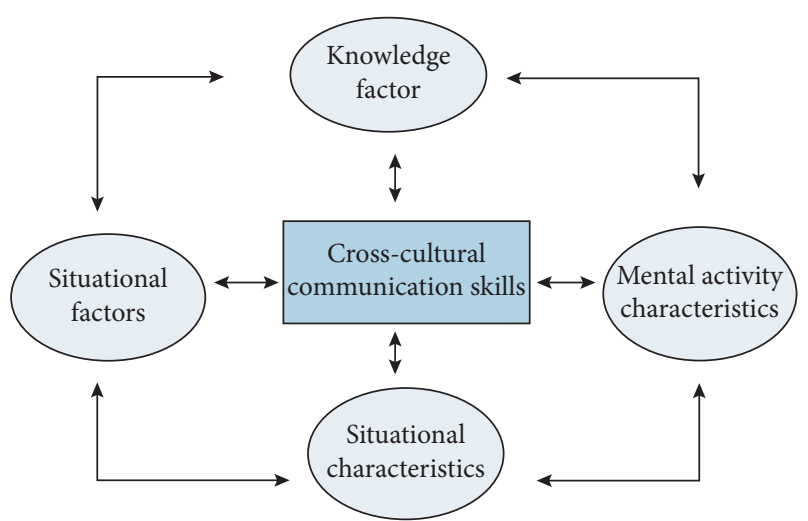

Figure 1: Cross-cultural communication elements of tourism English talents.

mean the real context of cross-cultural communication. The situational characteristics affecting their communicative competence are environmental context, prior contact, status differences, and third-party interference. Therefore, individuals have increased knowledge and experience in crosscultural communication, and, as a result, their motivation has increased and they have actively participated in communicative activities, and vice versa, forming a learning cycle of good success.

\subsection{The Elements of Cross-Cultural Communicative Compe-} tence of Tourism English Talents. The cross-cultural communicative competency elements constitute the first element of the domestic study's cross-cultural communicative competencies and are summarized into four communicative competence systems: basic communicative competence systems, emotional and relational competence systems, plot competence systems, and strategic capacity system. The elements of cross-cultural communicative competence of tourism English talents are shown in Figure 2.

From Figure 2, basic communicative competence system refers to the communicative ability that an individual needs to have in order to achieve communicative purposes, including language competence, and wants to connect with social cultural norms. These include verbal skills, nonverbal abilities, cultural abilities, interpersonal skills, and cognitive abilities, where the tourism English talent cultivation model believes that emotional competence refers to the communicator's recognition and understanding of the other party. Empathy needs to recognize the differences between individuals and cultures fully understand the self, suspend the self, eliminate the separation of oneself from the environment, conceiving for others, and finally preparing for empathy go through these six stages. Relational ability refers to the communication strategies used by the communicating parties in the process of communication. For example, the need for mutual communication between the two parties should be the basis for establishing relationships. The generation of consensus is the prerequisite for communication, and it is also related to cultural orientation and values.

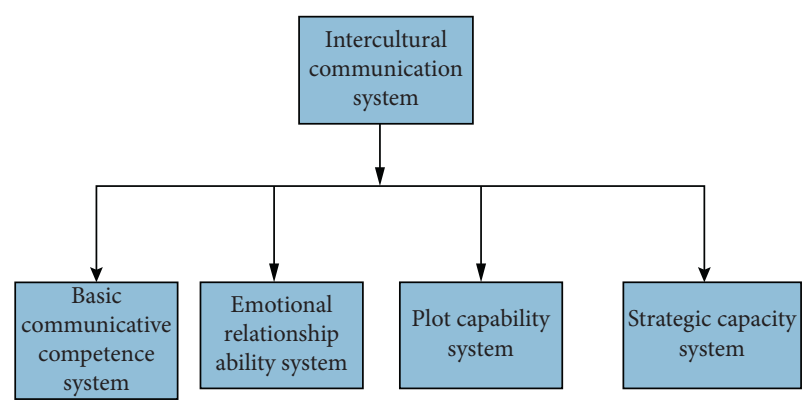

FIgURE 2: The elements of cross-cultural communicative competence of tourism English talents.

Similarly, the plot ability system comes to the plot competence system; it generally refers to the fixed sequence of interactions in a particular cultural environment. There are unique verbal and nonverbal rules in each plot. It proposes four aspects of ability, that is, common sense in communication, and also refers to the ability of a script communicator who directs behaviors to strive to achieve communicative purposes. The ability to follow the rules of communication in a particular context is appropriate to handle social scene responses. Moreover, strategy capability system proposed by the tourism English talent training model is different from the general communication strategy, but it is the remedial measures adopted by the communicators in the face of language barriers or verbal skills and clearly shows that strategic competence is an important part of the communicative competence.

\section{Big Data Feature Extraction}

In this section, we elaborate the big database model of tourism English talent resources distribution and the principles of big data mining for big-scale tourism English talent resources distribution. The feature fusion of tourism English talent resources optimized by big data fusion has good information mining capabilities.

3.1. Big Database Model of Tourism English Talent Resources Distribution. The big data characteristic information of tourism English talent resources distribution is usually expressed as a set of nonstationary broadband time series. The big-scale tourism English talent database big data resource information mining and scheduling is realized by using the method of time series detection [8]. Firstly, a big database model of tourism English talent resource distribution is constructed. In the construction of big data flow model of tourism English talent resource distribution, the task code is distributed to a number of data nodes by using distributed code execution mode, and the result is returned to the client after execution. It can effectively avoid the movement and transmission of cached data in the big database of tourism English talent resources distribution and use single computer or network computer to access the big amount of data and realize the evaluation and prediction of 
tourism resources information amount. The big database of tourism English talent resources distribution uses I/O, USB, and disk and other devices to achieve efficient read-write access [9]. Thus, the access and management model of tourism English talent resources distribution database is shown in Figure 3.

According to the analysis of Figure 2, suppose that the big database data set of tourism English talent resources distribution is the number of big data sets $X=\left\{x_{1}, x_{2}, \ldots, x_{n}\right\}$ of tourism English talent resources distribution, and the joint distribution feature vectors in $n$ are all $N$-dimensional vector $X$ containing $c$ big data classes of resource distribution. The clustering center of the big data class of $v_{i}=\left\{v_{i 1}, v_{i 2}, \ldots, v_{i p}\right\}$ resource distribution is big data. If the joint distribution function of nonstationary time sampling $\left\{x\left(t_{1}\right), \ldots, x\left(t_{n}\right)\right\}$ and the joint distribution function of $\left\{x\left(t_{1}+\tau\right), \ldots, x\left(t_{n}+\tau\right)\right\}$ are autocorrelation in the big database of tourism English talent resources distribution, the big data features of tourism English talent resource distribution are expressed as follows:

$$
\theta_{1}(k+1)=\theta_{1}(k)-\mu \operatorname{Re}\left[y(k) \varphi^{*}(k)\right] .
$$

The spectral features of the big database of tourism English talent resources distribution are analyzed by using aliasing spectrum ambiguity function, and the function relationship between the response variable and the forecast variable $\mathrm{X}=\left(\mathrm{x}_{1}, \ldots, \mathrm{x}_{n}, \ldots, \mathrm{x}_{n+m}\right)$ is determined. $\left\langle R_{1},+, \times\right\rangle$ and $\left\langle R_{2}, \oplus, \otimes\right\rangle$ are closed loop of the two eigenvector sets. Because the big data features of tourism English talent resource distribution database are combined with probability distribution function mapping $f: R_{1} \mapsto R_{2}$, for any $m_{i} \in R_{1}$, the big data information feature extraction equation of tourism English talent resource distribution can be obtained as follows:

$$
\begin{aligned}
& \dot{x}=V \cos \theta \cos \psi_{V}, \\
& \dot{y}=V \sin \theta, \\
& \dot{z}=-V \cos \theta \sin \psi_{V}, \\
& \dot{\vartheta}=\omega_{y} \sin \gamma+\omega_{z} \cos \gamma, \\
& \dot{\psi}=\frac{\left(\omega_{y} \cos \gamma-\omega_{z} \sin \gamma\right)}{\cos \vartheta}, \\
& \dot{\gamma}=\omega_{x}-\tan \vartheta\left(\omega_{y} \cos \gamma-\omega_{z} \sin \gamma\right),
\end{aligned}
$$

where $x, y$, and $z$ indicate the initial frequency of big data in the big database of tourism English talent resources distribution, $\psi_{V}$ indicates the big data training set of tourism English talent resources distribution, $\theta$ denotes the solution vector $Q$ in the space of the talent resource distribution variable, $\vartheta$ is the instantaneous amplitude of the complex signal $z(t)$ of the big tourism English talent resource distribution database, and $\gamma$ represents the statistical characteristics in the big tourism English talent database [10].

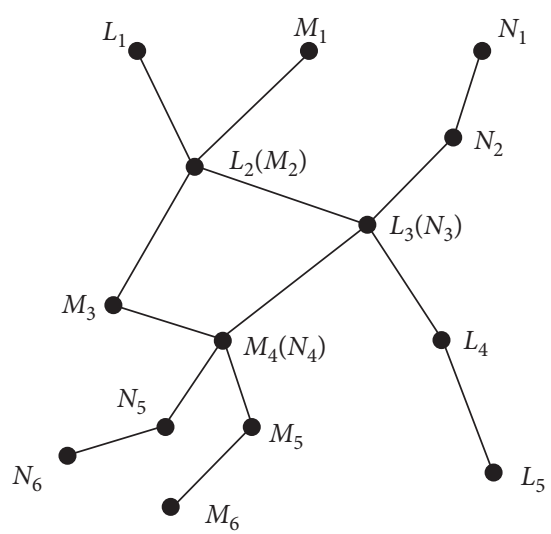

FIgURE 3: Distribution structure model of tourism English talent resources.

3.2. Principles of Big Data Mining. Based on constructing the distributed data structure model of tourism English talent training resources, the big data mining process is carried out and the big data distributed structure model of tourism time series algorithm provides regression algorithms that are used for optimizing and forecasting of continuous values like sales, overtime, and temperature. This algorithm can predict the trends that are based only on the original data set that is used to create a model. English talent resources distribution is designed. The computer data set under the condition of source distribution big data is fused and analyzed [11]. The schematic block diagram of big data mining for big-scale tourism English talent resources distribution is shown in Figure 4.

Fast data and resource information mining and scheduling are carried out in the big database of tourism English talent resources distribution to realize the integration of resource distribution big data [12]. Let $R$ be the trust relation with quaternion $\left(E_{i}, E_{j}, d, t\right)$ in the big data feature data of big-scale tourism English talent resources distribution. The information state equation of data classification attribute $A=\left\{A_{1}, A_{2}, \ldots, A_{m}\right\}$ and the information state equation of big data feature data integration of big-scale tourism English talent resources distribution are obtained as follows:

$$
\begin{aligned}
& P_{i}(t)=\sum_{n=1}^{N} \frac{A}{r} e^{-j k r} R_{i n} \frac{1}{r} e^{-i k r}, \\
& P_{i}(t)=\frac{A}{r^{2}} \sum_{n=1}^{N} e^{-j 2 k r} a_{i n} e^{j \psi_{i n}} .
\end{aligned}
$$

The time-frequency characteristics of the big database signal of tourism English talent resource distribution are calculated, and the big data structural model of the talent training information base resource distribution is constructed [13]. The big data model of resource distribution is obtained as follows:

$$
x(t)=\sum_{i=0}^{p} a\left(\theta_{i}\right) s_{i}(t)+n(t) .
$$




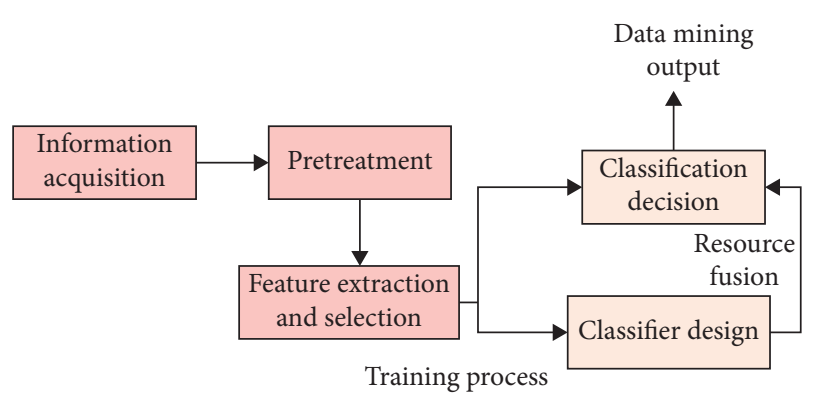

Figure 4: Principles of big data mining for big-scale tourism English talent resources distribution.

The big data characteristic data of tourism English talent resource distribution are sampled and updated in sequence, and the spectrum of nonstationary broadband signal $z(t)$ in the big database of tourism English talent resource distribution is obtained. Furthermore, time-frequency analysis is used to carry out transient analysis [14]. According to the estimation of time frequency, the probability function density expression of the accuracy of big data characteristic data for the distribution of big tourism English talent resources is as follows:

$$
p(y \mid \alpha, \theta)=\sum_{k=1}^{K} \alpha_{k} p_{k}\left(y \mid \mu_{k}, \sum k\right) .
$$

Big tourism English talent resource distribution big data is a sample set composed of $n$ samples. There are $m$ indexes in each big data sample of tourism English talent resource distribution; then the index characteristic vector of $j$ sample is $x_{j}=\left\{x_{1 j}, x_{2 j}, \ldots, x_{m j}\right\}^{T}$, tourism English. The a posteriori probability of big data is estimated as $p\left(x_{0}\right)$ :

$$
P_{i j}(k)=\frac{\left(l_{j}(k)-l_{i}(k)\right) \eta_{i j}(k)}{\sum_{j \in N_{i}(k)}\left(l_{j}(k)-l_{i}(k)\right) \eta_{i j}(k)} .
$$

According to the results of big data mining, data sharing and resource scheduling in the process of talent training are carried out by combining association feature mining and information fusion methods.

\subsection{Optimization of Cross-Cultural Communicative} Competence. Constructing English for tourism in the context of big data is not exactly equivalent to the Basic English content. It has its uniqueness, except listening, speaking, reading, writing, and translation of Basic English training. In addition to their abilities, they must also develop their professional English skills, relevant cross-cultural thinking skills, and communication skills. Therefore, in the tourism English curriculum, student's cross-cultural communication skills should be cultivated to meet the requirements of the current society, disciplines, and students themselves. From the study of intercultural communicative competence by various scholars at home and abroad, crosscultural communicative competence is a comprehensive, multidimensional system such as knowledge system, relationship system, and behavior system. Based on these researches on the elements of intercultural communication, and combining the characteristics of the curriculum of tourism English under the big data environment, a crosscultural communicative competence framework for tourism English teaching in the context of big data is constructed. The framework of cross-cultural communicative competence of English tourism talents is shown in Figure 5.

From Figure 5, we can observe that, for a student majoring in tourism management, English communication skills have become a core skill. Compared with other professional students, English communication ability is a requirement within the category of professional competence. Therefore, colleges and universities pay great attention to tourism English teaching in the context of big data. However, in practice, students find that they have a high level of enthusiasm for learning professional English, but the results are not obvious. The bigger reason is that travel professional English is different. Basic English requires global awareness and industry awareness, which is the basis for developing cross-cultural communication skills. The global awareness mentioned here includes cross-cultural awareness and crosscultural thinking. Cross-cultural awareness can also be called cultural awareness [15]. Similarly, In the process of crosscultural communication, cultural conflicts occur everywhere. In order to shorten the conflict period, it is necessary to adapt to the rapid recovery period. This is a process of entering a steady state through a series of adjustments from a relatively unstable state [16]. The theory of adaptation to cross-cultural adaptation process can be summarized as a model curve [17]. The adaptability plays an important role in the exchange process. This is a turning point from uncertainty decision rather than acceptance. Adaptability includes two elements: psychological adaptability and cultural adaptability $[18,19]$.

Moreover, in the reform of foreign language classroom teaching, cultivating student's communicative application ability is the focus of foreign language circles in recent years. The University English Classroom Requirements with a target meaning also highlights language knowledge and application capabilities. For the purpose of tourism English, language knowledge and cultural knowledge are the basic teaching contents of the teacher's class. In addition, the ability of the first three media is in communication practice. Communicative practice ability is the highest level of intercultural communication ability. It includes communicative competence, nonverbal communication, and communication skills. The communicative practice ability emphasizes the use of linguistic, nonverbal, and related strategies in practical situations to solve practical cross-cultural communication problems. The ability of a large communication strategy is the core competitiveness of tourism services. 


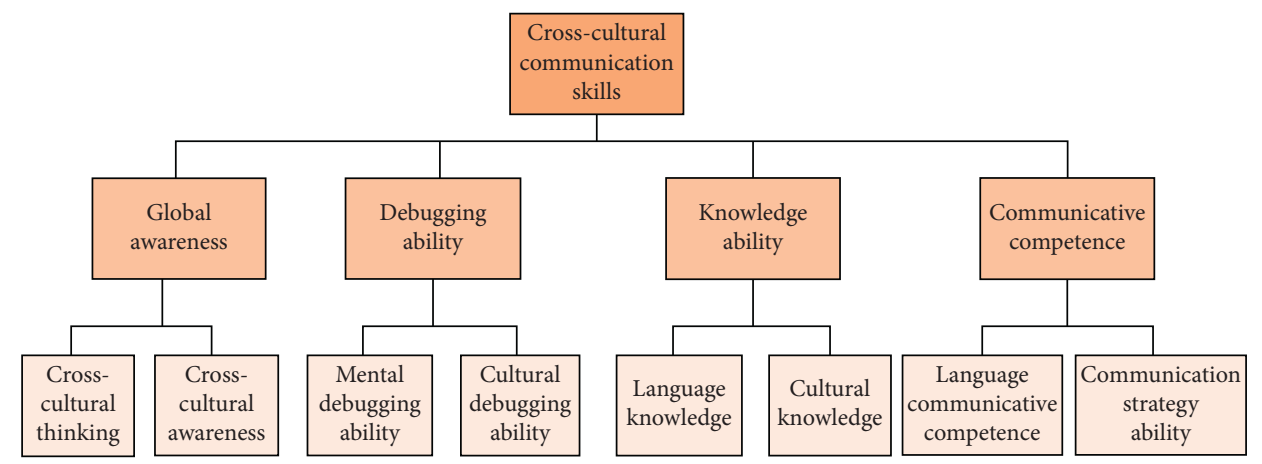

FIGURE 5: Framework of cross-cultural communicative competence of English tourism talents.

3.4. Feature Classification and Information Fusion of Tourism English Talent Resources. The fuzzy clustering method is used to deal with the adaptive clustering of tourism English talent resources distribution, the attribute distribution structure model is used to classify the features and information fusion of tourism English talent resources, and the time-frequency analysis method is adopted. By extracting the big data feature of resource distribution, the information state function of big data feature data of tourism English talent resource distribution is presented as follows:

$$
\begin{aligned}
\dot{x}_{i} & =f_{i}\left(x_{i}, u_{i}\right) D\left(x_{i}, A_{j}(L)\right) \\
& =\min \left\{D\left(x_{i}, A_{j}(L)\right)\right\},
\end{aligned}
$$

where $x_{i} \in R^{n}$, and the state vector of big data feature data representing the distribution of tourism English talent resources is presented. Based on the above analysis, the feature extraction of resource distribution big data in the big database of tourism English talent resources distribution is realized, and the big scale is extracted. The big data training sample of tourism English talent resource distribution in the database, the time-frequency feature of the extracted tourism English talent resource distribution big data is rearranged by sliding time window, and the tourism English talent database distribution big data is carried out. The expression of the frequency domain model is as follows:

$$
x_{n}=[x(0), x(1), \ldots, x(N-1)]^{T} .
$$

The big data fusion algorithm of tourism English talents training is improved by phase spectrum compensation, and the attribute weights of random linear access channel for resource big data fuzzy decision fusion of tourism English talent training are obtained as follows:

$$
F=\left[\begin{array}{ccccc}
\omega^{0} & \omega^{0} & \omega^{0} & \cdots & \omega^{0} \\
\omega^{0} & \omega & \omega^{2} & \cdots & \omega^{k-1} \\
\omega^{0} & \omega^{2} & \omega^{4} & \cdots & \omega^{2(k-1)} \\
\vdots & \vdots & \vdots & \vdots & \vdots \\
\omega^{0} & \omega^{k-1} & \omega^{2(k-1)} & \cdots & \omega^{(k-1)^{2}}
\end{array}\right]=\prod_{0 \leq j<i \leq k-1}\left(\omega^{i}-\omega^{j}\right) .
$$

The vector model structure of big data fuzzy decision fusion for tourism English talents training is expressed as follows:

$$
\left\{\begin{array}{l}
\dot{m}_{i}(t)=-a_{i} m_{i}(t)+b_{i}\left(p_{1}(t-\sigma), p_{2}(t-\sigma), \ldots, p_{n}(t-\sigma)\right), \\
\dot{p}_{i}(t)=-c_{i} p_{i}(t)+d_{i} m_{i}(t-\tau) .
\end{array}\right.
$$

Combined with the association feature mining and information fusion method, data sharing and resource scheduling are carried out in the training process, so that the data fusion capacity is improved $[15,16]$.

3.5. Big Data Fusion and Optimization Mining of English Talent Resource Distribution. In the tourism English talent database, the prediction of packet information can be as follows: $\left\{\lambda_{i}: 1 \leq i \leq S\right\}$, criterion $\left\{R_{j}: 1 \leq j \leq L\right\}$, and density prior information sliding time window rearrangement of big data for tourism English talent resources distribution calculation is expressed as follows: 


$$
\begin{aligned}
N_{i, j}= & \left(f(A)_{i, 1} \otimes f(B)_{1, j}\right) \oplus\left(f(A)_{i, 2} \otimes f(B)_{2, j}\right) \oplus \cdots \oplus\left(f(A)_{i, h} \otimes f(B)_{h, j}\right) \\
= & \left(\left(K_{A} \cdot\left(A_{i, 1}+r p\right) \cdot k_{A}^{r} \bmod n\right) \cdot\left(K_{B} \cdot\left(B_{1, j}+r p\right) \cdot k_{B}^{r} \bmod n\right)\right) \oplus \cdots \oplus \\
& \left(\left(K_{A} \cdot\left(A_{i, h}+r p\right) \cdot k_{A}^{r} \bmod n\right) \cdot\left(K_{B} \cdot\left(B_{h, j}+r p\right) \cdot k_{B}^{r} \bmod n\right)\right) \\
= & \sum_{u=1}^{h}\left(\left(K_{A} \cdot\left(A_{i, u}+r p\right) \cdot k_{A}^{r} \bmod n\right) \cdot\left(K_{B} \cdot\left(B_{u, j}+r p\right) \cdot k_{B}^{r} \bmod n\right)\right) \bmod n \\
= & \sum_{u=1}^{h}\left(\left(\frac{s \cdot l}{k_{A}^{r}} \cdot\left(A_{i, u}+r p\right) \cdot k_{A}^{r} \bmod n\right) \cdot\left(\frac{s \cdot l}{k_{B}^{r}} \cdot\left(B_{u, j}+r p\right) \cdot k_{B}^{r} \bmod n\right)\right) \bmod n \\
= & (s \cdot l)^{2} \sum_{u=1}^{h}\left(\left(A_{i, u}+r p\right) \cdot\left(B_{u, j}+r p\right)\right) \bmod n \\
= & (s \cdot l)^{2} \sum_{u=1}^{h}\left(A_{i, u} \cdot B_{u, j}+\left(A_{i, u}+B_{u, j}\right) \cdot r p+r^{2} p^{2}\right) \bmod n .
\end{aligned}
$$

It can be seen that the nearest-neighbor matching method using the time-frequency eigenvector can find out the potential matching of the big data features of the tourism English talent resources:

$$
\begin{aligned}
& \left(V\left(a_{1}, \ldots, a_{m}\right)^{\left(\alpha_{1}, \ldots, \alpha_{m}\right)}\right)^{-1} V\left(b_{1}, \ldots, b_{m}\right)^{\left(\beta_{1}, \ldots, \beta_{m}\right)} \\
& =\left(\left(V\left(a_{1}, \ldots, a_{m}\right)^{-1} V\left(b_{1}, \ldots, b_{m}\right)\right)^{\left(\alpha_{1}^{-1}, \ldots, \alpha_{m}^{-1}\right)^{T}}\right)^{\left(\beta_{1}, \ldots, \beta_{m}\right)} .
\end{aligned}
$$

The fuzzy feature balance metric of the differences between $j$ and $c$ categories in the big data sample of tourism English talent resources distribution can be expressed as follows:

$$
f_{j}\left(u_{j}, s, w\right)=\sum_{h=1}^{c}\left\{u_{h j}^{2}\left[\sum_{i=1}^{m}\left[w_{i}\left(r_{i j}-s_{i h}\right)\right]^{2}\right]^{\alpha / 2}\right\} .
$$

The big data signal analysis of tourism English talent resources distribution is extended to the disturbed environment. For the time series of tourism English talent resources $\left\{x\left(t_{0}+i \Delta t\right)\right\}, i=0,1, \ldots, N-1$, the fast resource information of the big database of tourism English talent resources distribution is established. The mining and scheduling model objective function is,

$$
\min \left\{f(u, s, w)=f_{1}\left(u_{1}, s, w\right), f_{2}\left(u_{2}, s, w\right), \ldots, f_{n}\left(u_{n}, s, w\right)\right\} .
$$

\section{Result Analysis and Discussion}

In this section, we compare the performance and utility of the proposed model. The efficiency of the proposed model was initially tested using the optimization of tourism English talent training mode is realized. In order to improve the ability of fast resource information mining and scheduling, a sliding time window rearrangement of big data prior information of tourism English talent resource distribution in big tourism English talent database is carried out.

4.1. Experimental Setup. In this paper, each algorithm was evaluated through experiments, and broad data computational approaches were used, that is, the open-source Apache Hadoop and the Apache Spark platform. First of all, we created a Spark cluster that uses physical nodes, that is, 80 nodes. To determine the basic software and hardware specifications for the experiment, all computing nodes have been simplified, and their configuration is shown in Table 1 . For example, operating systems Ubuntu 18, Spark 3.01, and Hadoop 3.2 are used in experimental configurations. In all 80 nodes, one node was used as a master node or as a processing node, and the remaining 79 nodes were used as slaves in the cluster. Apache Hadoop's software library is a tool used in basic programming models to spread vast data collection through computer clusters. For each local server calculation and storage, it is scheduled to reach multiple computers. Apache Spark is a hierarchical programming engine for large-scale data processing that provides highlevel APIs for Java, Python, R, and Scala and is used as an advanced map driver. Apache Spark enables a wide range of high-level tools, including SQL Spark for structured data analysis, MLlib for apprenticeships, and GraphX for graphics processing.

4.2. Simulation Experiment and Result Analysis. In order to test the application performance of this algorithm in realizing the big data mining of tourism English talent resource distribution and the optimization of training mode, the simulation experiment is carried out. In the experiment, 100 pieces of big data sample data of tourism English talent resource distribution are selected, the number of talent attribute samples is 50 , and the dimension of time-frequency 
TABle 1: Configuration detail of cluster.

\begin{tabular}{ccc}
\hline \multirow{4}{*}{ Hardware } & Number of cluster nodes & 80 \\
& Hard disk & $500 \mathrm{~GB}$ \\
& No. of CPU cores & 8 \\
& Processor & $3.0 \mathrm{GHz} \times 4$ \\
& Connectivity & $100 \mathrm{Mbps}$ ethernet LAN \\
& RAM size & $30 \mathrm{~GB}$ \\
Software & Data block size & $256 \mathrm{Mb}$ \\
& Operating system & Ubuntu 18 \\
& Java-JDK & $\mathrm{V}-1.8$ \\
& Hadoop & $\mathrm{V}-3.0$ \\
& Spark & $\mathrm{V}-3.0 .1$ \\
& Open-PDC & $\mathrm{V}-1.5$ \\
& Python & $\mathrm{V}-3.8$ \\
\hline
\end{tabular}

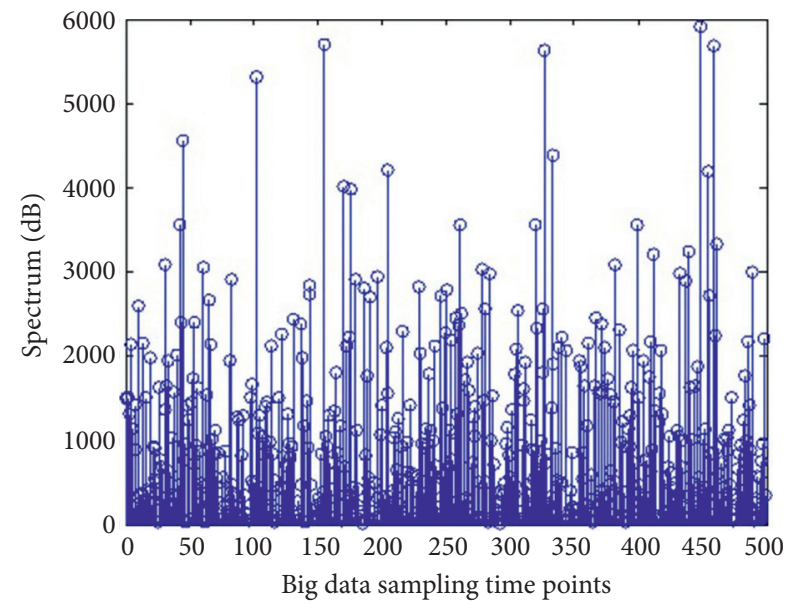

FIGURE 6: Big data mining results of tourism English talent training.

feature state space of the big database of tourism English talent resource distribution is 10, which is sampled according to the characteristic data. According to the above simulation experimental environment design and parameter design, the big data feature extraction and resource information mining of tourism English talent resource distribution are carried out, and the data mining is obtained. The effect of some parameters, that is, learning rate and activation functions, on accuracy is summarized in Table 1, that is, performance of different phenotype. We have optimized the parameters settings for all the learning algorithms for accuracy-based analysis. The result is shown in Figure 6.

The results of the analysis of Figure 6 show that the data mining of tourism English talent training resource distribution using this method is of high accuracy. The fast resource information mining of big data for tourism English talent resources distribution can be realized. SQL Server Data Mining offers Office 2007 data mining additives for the detection of data patterns and relationships. This is also helpful in improving the analysis. The add-in called Data Mining Client for Excel is used first to plan, compile, analyze, handle, and forecast data. In order to compare the performance of scheduling, different methods are used to mine the big data of tourism English talent

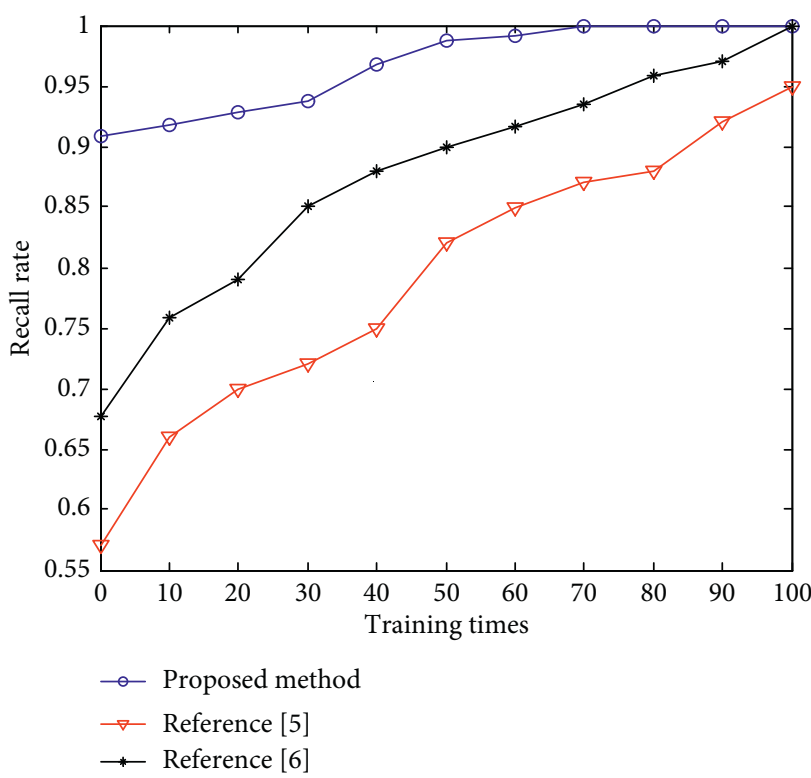

FIgURE 7: Big data analysis performance test.

resources distribution, and the recall test results are shown in Figure 7.

The results of the analysis in Figure 7 show that this method has good accuracy and big data clustering ability for tourism English talent resource data mining, which can effectively guide the construction of a new model for tourism English talent training.

\section{Conclusions}

In this paper, a new model of tourism English talent training based on big data is proposed, and the big data mining model of tourism English talents is constructed. Traditional methods use basic algorithms to predict the future. However, it does not provide reliable findings as compared to data mining. In the cloud storage system, the optimal scheduling design of tourism English talent training resource big data is carried out, and the fuzzy clustering method is used to deal with the adaptive clustering of tourism English talent resource distribution big data.

Moreover, the attribute distribution structure model is constructed to classify and fuse the features of tourism English talent resources, and the methods of association feature mining and information fusion are combined to share data and schedule resources in the process of talent training. Time-frequency features of tourism English talent resource distribution big data are extracted to realize the fast mining and positioning of the data. The simulation results show that this method has good accuracy and big data clustering ability, which can effectively guide the construction of a new model of tourism English talent training. This model has a good application value in promoting the promotion of tourism English talent training model. In the future, big data research needs to deal with the relationship between data and theory. The combination of data driving 
and theoretical driving is conducive to the benign development of the mutual promotion of theory and data.

\section{Data Availability}

The data used to support the findings of this study are included within the article.

\section{Conflicts of Interest}

The author declares that there are no conflicts of interest regarding the publication of this paper.

\section{Acknowledgments}

The paper was supported by Anhui Province Overseas Study Visiting Project of Universities Excellent Talents Cultivation in 2017 (Grant no. GXFX2017237); Internet-Based Teaching Management Model Innovation Research, Anhui Vocational and Adult Education Association Education Scientific Research Planning Project (Grant no. AZJXH17156); Provincial Quality Engineering Practical English Excellent Open Course of Anhui Provincial Education Department (Grant no. 2017KFK136); Research on the Professional Development of Public English Teachers from the Perspective of Innovative Development of Higher Vocational Education, Key Project of Humanities and Social Sciences in Universities of Anhui Province (Grant no. SK2018A0832); Research on the Construction and Development of " $2+2+2$ " Practical English Mixed Teaching Curriculum System in Higher Vocational Colleges (Grant no. SK2018A1170); Provincial Quality Engineering Teaching Research Project of Anhui Provincial Department of Education: Higher Vocational English Teaching Research Based on EOP + PE Ability Cultivation from NA Perspective (Grant no. 2019JYXM0613); and Provincial Quality Engineering Teaching Research Project of Anhui Provincial Education Department in 2020: Study on Practical English Course Ideological and Political Construction from the Perspective of "Taoism" (Grant no. 2020KCSZYJXM228).

\section{References}

[1] T.-z. Wen, A.-q. Xu, and G. Chneg, "Multi-fault diagnosis method based on improved ENN2 clustering algorithm," Control and Decision, vol. 30, no. 6, pp. 1021-1026, 2015.

[2] A. Kumar, R. Pooja, and G. K. Singh, "Design and performance of closed form method for cosine modulated filter bank using different windows functions," International Journal of Speech Technology, vol. 17, no. 4, pp. 427-441, 2014.

[3] N. Rajapaksha, A. Madanayake, and L. T. Bruton, "2D spacetime wave-digital multi-fan filter banks for signals consisting of multiple plane waves," Multidimensional Systems and Signal Processing, vol. 25, no. 1, pp. 17-39, 2014.

[4] Y. Jiang, F. L. Chung, S. Wang, Z. Deng, J. Wang, and P. Qian, "Collaborative fuzzy clustering from multiple weighted views," IEEE Transactions on Cybernetics, vol. 45, no. 4, pp. 688-701, 2015.

[5] A. Bi and S. Wang, "Transfer affinity propagation clustering algorithm based on kullback-leiber distance," JEIT, vol. 38, no. 8, pp. 2076-2084, 2016.
[6] B. N. Alahmad and S. Gopalakrishnan, "Energy efficient task partitioning and real-time scheduling on heterogeneous multiprocessor platforms with QoS requirements," Sustainable Computing: Informatics and Systems, vol. 1, no. 4, pp. 314-328, 2011.

[7] H. Wang, H. Jin, J. Wang, and W. Jiang, "Optimization approach for multi-scale segmentation of remotely sensed imagery under k-means clustering guidance," Acta Geodaetica et Cartographica Sinica, vol. 44, no. 5, pp. 526-532, 2015.

[8] O. Shi-feng, Y. Gao, and Z. Xiao-hui, "Adaptive combination algorithm and its modified scheme for blind source separation," Journal of Electronics \& Information Technology, vol. 33, no. 5, pp. 1243-1247, 2011.

[9] G. Tian, H. E. Ke-qing, J. Wang et al., "Domain-oriented and tag-aided web service clustering method," Chinese Journal of Electronics, vol. 43, no. 7, pp. 1266-1274, 2015.

[10] W. Tao, C. Lifei, and G. Gongde, "High-dimensional data clustering algorithm with subspace optimization," Journal of Computer Applications, vol. 34, no. 8, pp. 2279-2284, 2014.

[11] Y. Lei, X. Yu, S. Yue et al., "Research on PSO-based intuitionistic fuzzy kernel clustering algorithm," Journal of Communication, vol. 4, no. 5, Article ID 2015099, 2015.

[12] B. Zhang, H. Jie, G. Ma et al., "Mixture of probabilistic canonical correlation analysis," Journal of Computer Research and Development, vol. 52, no. 7, pp. 1463-1476, 2015.

[13] C. Sun, C. Yang, S. Fan et al., "Energy efficient distributed clustering consensus filtering algorithm for wireless sensor networks," Information and Control, vol. 44, no. 3, pp. 379-384, 2015.

[14] X.-y Guo, "Simulation and analysis on uncertain attenuation property of underwater acoustic signal for oil field pipe," Computer Simulation, vol. 31, no. 3, pp. 118-121, 2014.

[15] W. Zhang and Q. Chen, "Network intrusion detection algorithm based on HHT with shift hierarchical control," Computer Science, vol. 41, no. 12, pp. 107-111, 2014.

[16] H. Luo, Y. Qu, and Y. Yu, "Oscillation criteria of second order neutral delay emden-fowler equations with positive and negative coefficients," Acta Mathematicae Applicatae Sinica, vol. 40, no. 5, pp. 667-675, 2017.

[17] Z. Duo, G. Xu, X. Chen, and K. Yuan, "Rational non-hierarchical quantum state sharing protocol," Computers, Materials \& Continua, vol. 58, no. 2, pp. 335-347, 2019.

[18] Q. He, S. Yu, H. Xu et al., "A weighted threshold secret sharing scheme for remote sensing images based on chinese remainder theorem," Computers, Materials \& Continua, vol. 58, no. 2, pp. 349-361, 2019.

[19] F. Guo, P. Liu, W. Ren et al., "Research on the relationship between garlic and young garlic shoot based on big data," Computers, Materials \& Continua, vol. 58, no. 2, pp. 363-378, 2019. 